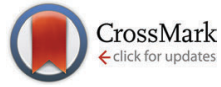

Cite this: Phys. Chem. Chem. Phys., 2015, 17, 11140

Received 6th March 2015, Accepted 27th March 2015

DOI: $10.1039 / c 5 c p 01336 d$

www.rsc.org/pccp

\section{Optical writing and reading with a photoactivatable carbazole $\dagger$}

\author{
Jaume Garcia-Amorós, ${ }^{\star a}$ Subramani Swaminathan, ${ }^{a}$ Yang Zhang, ${ }^{a}$ Santi Nonell ${ }^{\mathrm{b}}$ \\ and Françisco M. Raymo*a
}

The fluorescence of a carbazole chromophore can be activated irreversibly under optical control with the photoinduced opening of an oxazine ring. In proximity to silver nanoparticles, the quantum efficiency of this photochemical transformation and that of the emissive process increase significantly. The plasmonic effects responsible for such enhancements, together with the photochemical and photophysical properties engineered into this particular photoactivatable fluorophore, permit the optical writing and reading of microscaled patterns at low illumination intensities.

The photochemical conversion of a nonemissive reactant into a fluorescent product permits the activation of fluorescence under optical control. ${ }^{1-5}$ Specifically, illumination of the reactant at an appropriate activation wavelength $\left(\lambda_{\mathrm{Ac}}\right)$ induces the photochemical transformation and irradiation of the resulting product at a given excitation wavelength $\left(\lambda_{\mathrm{Ex}}\right)$ generates fluorescence. Under these conditions, the interplay of two lasers operating at $\lambda_{\mathrm{Ac}}$ and $\lambda_{\mathrm{Ex}}$ can be exploited to switch fluorescence on within a defined region of space at a specific interval of time. In turn, such spatiotemporal control allows the monitoring of dynamic processes in real time with the sequential acquisition of fluorescence images after activation. ${ }^{6,7}$ Additionally, it also enables the reconstruction of fluorescence images with subdiffraction resolution after reiterative activation and excitation sequences. ${ }^{8,9}$ As a result, photoactivatable fluorophores are becoming invaluable probes for the investigation of the dynamic and structural properties of a diversity of biological specimens and synthetic materials.

The illumination of metallic nanostructures at appropriate wavelengths results in the coupling of incident radiations and surface plasmons to produce a local enhancement in

\footnotetext{
${ }^{a}$ Laboratory for Molecular Photonics, Department of Chemistry, University of Miami, 1301 Memorial Drive, Coral Gables, Florida, 33146-0431, USA. E-mail: jgarciaamoros@ub.edu,fraymo@miami.edu

${ }^{b}$ Grup d'Enginyeria Molecular, Institut Quimic de Sarrià, Universitat Ramón Llull, Via Augusta 390, E-08017, Barcelona, Spain

$\dagger$ Electronic supplementary information (ESI) available: Experimental procedures, voltammograms, absorption and emission spectra, fluorescence images, atomic force microscopy (AFM) images, fluorescence decays. See DOI: 10.1039/c5cp01336d
}

electromagnetic field. ${ }^{10-12}$ This phenomenon facilitates electronic transitions in proximal chromophores, relative to distal counterparts, to promote photochemical transformations or enhance fluorescence within relatively short distances from the nanostructures. In this context, the concatenation of a photochemical reaction (activation) with a photophysical process (fluorescence), responsible for the operating principles of photoactivatable fluorophores, is particularly valuable to engineer plasmonic effects into photoresponsive materials. In fact, both the activation of the reactant and the emission of the product can be facilitated under the influence of metallic nanostructures. Indeed, we relied on these effects to implement autocatalytic loops with photoactivatable anthracenes, ${ }^{13}$ enable two-photon activation at low illumination intensities with a photoactivatable carbazole ${ }^{14}$ and enhance the production and radiative decay of singlet molecular oxygen. ${ }^{15}$ In the wake of these precedents, we realized that the irreversible activation of fluorescence under plasmonic assistance could be exploited to generate and detect fluorescent patterns efficiently with light. In this article, we report the synthesis of a carbazoleoxazine dyad specifically designed for this application and the experimental implementation of such a mechanism for optical writing and reading.

Compounds 1 and 2 (Fig. 1) were isolated in yields of 9 and $27 \%$ respectively, after the condensation of aldehyde 3 with oxazine 4 and indolenine 5 respectively, in the presence of trifluoroacetic acid (TFA). Electrospray ionization mass spectra (ESIMS) together with ${ }^{1} \mathrm{H}$ nuclear magnetic resonance (NMR) spectra confirmed their structural identities.

The absorption spectrum (a in Fig. 2) of 1 shows an intense band at $285 \mathrm{~nm}$ for the carbazole chromophore. Illumination at a $\lambda_{\text {Ex }}$ positioned within this absorption does not cause any detectable fluorescence. Electron transfer from the excited carbazole to the adjacent 4-nitrophenoxy group is presumably responsible for preventing radiative deactivation. In fact, the oxidation potential $(+0.92 \mathrm{~V})$ of 9-methyl-9H-carbazole ( 7 in Fig. S1, ESI $\dagger)$, the reduction potential $(-1.33 \mathrm{~V})$ of $\mathbf{4}$ and the energy $(3.32 \mathrm{eV})$ of the first singlet excited state of 7 , suggest this process to be exergonic with a free-energy change of $-1.1 \mathrm{eV}$. 


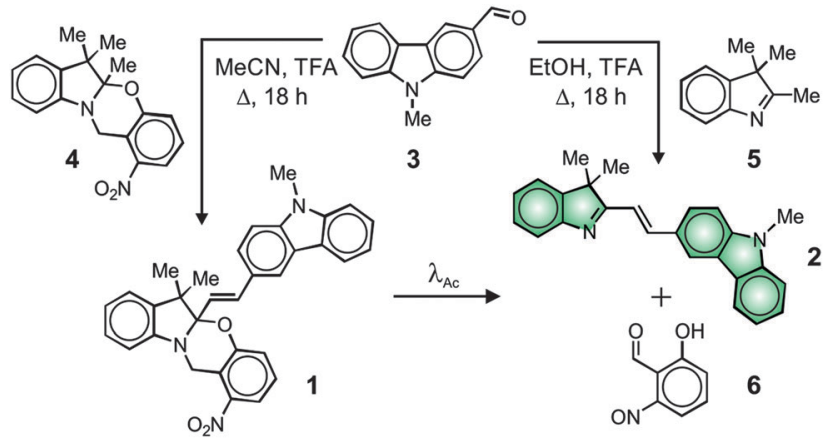

Fig. 1 Synthesis of $\mathbf{1}$ and $\mathbf{2}$ and photochemical transformation of $\mathbf{1}$ into $\mathbf{2}$ and 6 .

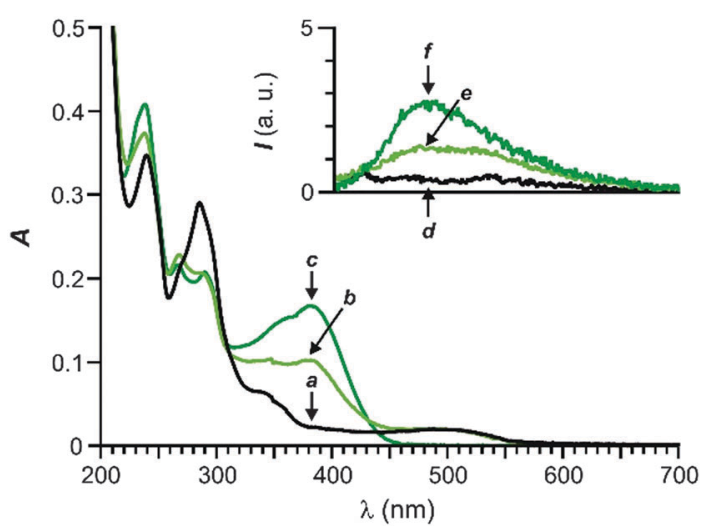

Fig. 2 Absorption (a-c) and emission ( $d-f, \lambda_{E x}=380 \mathrm{~nm}$ ) spectra of $\mathrm{MeCN}$ solutions $\left(10 \mu \mathrm{M}, 20^{\circ} \mathrm{C}\right)$ of 1 , before (a and d) and after (b and e) irradiation $\left(\lambda_{\mathrm{AC}}=350 \mathrm{~nm}, 4.88 \mathrm{~mW} \mathrm{~cm}^{-2}, 10 \mathrm{~min}\right)$, and of 2 (c and f).

Illumination of 1 at a $\lambda_{\mathrm{Ac}}$ of $350 \mathrm{~nm}$ cleaves its 2-nitrobenzyl group to produce 2 and 6 (Fig. 1). Consistently, absorption and emission bands develop at 380 and $490 \mathrm{~nm}$ (b and e in Fig. 2) respectively, during the photolytic transformation. These bands resemble those observed in the spectra ( $c$ and $f$ in Fig. 2) of 2 and confirm the photoinduced formation of this species. The fluorescence quantum yield $(\phi)$ of 2 , however, is as low as 0.02 and only a relatively weak emission develops after photolysis of 1 (d and e in Fig. 2). Nonetheless, time-correlated single-photon counting (TCSPC) experiments confirm the assignment of the photogenerated band to 2. Specifically, the emission intensity, detected after photolysis of $\mathbf{1}$, decays biexponentially with lifetimes ( $\tau_{1}$ and $\tau_{2}$ in Table S1, ESI $\dagger$ ) of 0.65 and $3.6 \mathrm{~ns}$ (76 and $24 \%$ ). Essentially the same temporal fluorescence evolution is also observed for 2 with $\tau_{1}$ and $\tau_{2}$ of 0.94 and $3.8 \mathrm{~ns}$ (66 and 34\%). Presumably, the biexponential decay is a result of the coexistence of two emissive conformers in solution.

The square wave voltammogram (a in Fig. 3) of 1 shows peaks at -1.40 and $-1.86 \mathrm{~V}$ for the two consecutive monoelectronic reductions of the nitro group. ${ }^{16}$ After illumination at $\lambda_{\mathrm{Ac}}$, both peaks disappear with the concomitant appearance of new ones at -1.18 and $-2.08 \mathrm{~V}$ (b in Fig. 3). Literature data ${ }^{17,18}$ suggest that these additional peaks correspond to reductions of the nitroso and aldehyde groups of 6 respectively and confirm the photoinduced formation of this species.

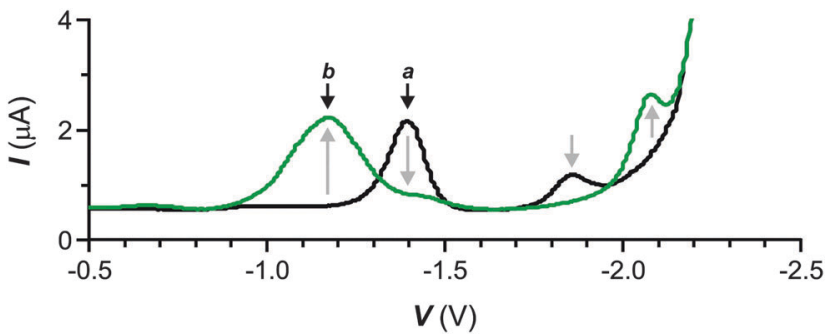

Fig. 3 Square wave voltammograms $\left[50 \mathrm{mV} \mathrm{s}^{-1}, \mathrm{~V} v \mathrm{~s} . \mathrm{Ag} \mathrm{Ag}^{+}(1 \mathrm{mM}\right.$ in $\left.\left.\mathrm{AgNO}_{3}\right), \mathrm{Bu}_{4} \mathrm{NPF}_{6}(50 \mathrm{mM})\right]$ of a MeCN solution of $1\left(100 \mu \mathrm{M}, 20^{\circ} \mathrm{C}\right)$ before (a) and after (b) illumination ( $\left.\lambda_{\mathrm{Ac}}=350 \mathrm{~nm}, 4.88 \mathrm{~mW} \mathrm{~cm}^{-2}, 10 \mathrm{~min}\right)$.

The photoinduced conversion of $\mathbf{1}$ into $\mathbf{2}$ and $\mathbf{6}$, observed in acetonitrile solutions, occurs also within poly(butylmethacrylate) (PBMA) matrices with similar spectral changes (Fig. S2, ESI $\dagger$ ). In PBMA, however, $\phi$ for 2 raises to 0.23 , while $\tau_{1}$ and $\tau_{2}$ for the corresponding biexponential decay drop to 0.15 and $1.1 \mathrm{~ns}$ (87 and 13\%). These trends suggest that the rigid polymer matrix accelerates the radiative deactivation of the excited state, while delaying its nonradiative decay. Presumably, the restricted rotation of the $3 \mathrm{H}$-indole and carbazole fragments, around the adjacent $[\mathrm{C}-\mathrm{C}]$ bonds, in the rigid environment is mostly responsible for this behavior. Essentially the same temporal fluorescence profile is detected also after illumination of a PBMA film, doped with $\mathbf{1}$, at $\lambda_{\mathrm{Ac}}$. Once again, the emission intensity decays biexponentially with $\tau_{1}$ and $\tau_{2}$ of 0.15 and $1.1 \mathrm{~ns}$ (87 and 13\%), confirming that the fluorescent species photogenerated in the polymer matrix is indeed 2.

Fluorescence images of doped PBMA films further confirm the photoinduced formation of the fluorescent product. Specifically, illumination of an ellipsoidal region at a $\lambda_{\mathrm{Ac}}$ of $405 \mathrm{~nm}$ for $90 \mathrm{~s}$ converts 1 into 2 exclusively in the irradiated area. Comparison of images ( $a$ and b in Fig. 4 and Fig. S3, ESI $\dagger$ ), acquired by irradiating the entire field of view at the same wavelength $\left(\lambda_{\mathrm{Ex}}=\lambda_{\mathrm{Ac}}\right)$ for only $432 \mathrm{~ms}$ before and after activation, shows significant emission only in the activated area. Furthermore, the acquisition of an additional image (c in Fig. 4 and Fig. S3, ESI $\dagger$ ), 10 min after activation, does not reveal any noticeable change in the shape of the imprinted fluorescent pattern. Thus, the photogenerated fluorophores cannot diffuse within the rigid polymer matrix on this timescale. In turn, the lack of any diffusion offers the opportunity to induce and monitor the photochemical transformation within the very same area of the substrate. Specifically, the continuous
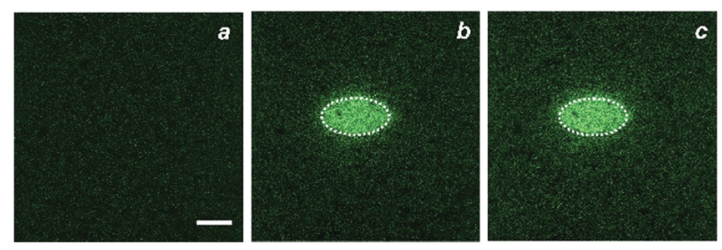

Fig. 4 Confocal laser-scanning fluorescence images $\left(\lambda_{E x}=405 \mathrm{~nm}, \lambda_{\mathrm{Em}}=\right.$ $430-650 \mathrm{~nm}$, scale bar $=30 \mu \mathrm{m})$ of a PBMA film, doped with $1(3 \% \mathrm{w} / \mathrm{w})$ and deposited on a glass slide with silver nanoparticles on its surface, recorded before (a), immediately (b) and $10 \mathrm{~min}$ (c) after illumination $\left(\lambda_{\mathrm{Ac}}=405 \mathrm{~nm}\right.$, $0.1 \mathrm{~mW}, 90 \mathrm{~s}$ ) of an ellipsoidal area within the imaging field. 


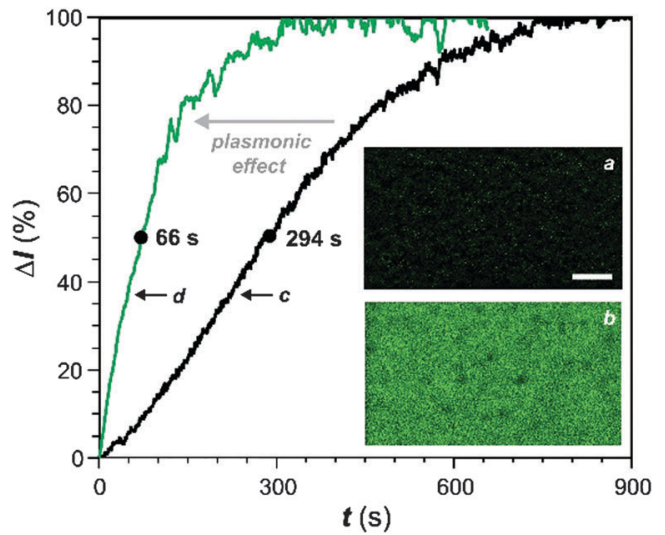

Fig. 5 Confocal laser-scanning fluorescence images $\left(\lambda_{E x}=405 \mathrm{~nm}, \lambda_{E m}=\right.$ $430-650 \mathrm{~nm}$, scale bar $=30 \mu \mathrm{m})$ of PBMA films, doped with $1(3 \% \mathrm{w} / \mathrm{w})$ and deposited on glass slides without (a) and with (b) silver nanoparticles on their surface, recorded after illumination ( $\lambda_{\mathrm{AC}}=405 \mathrm{~nm}, 0.1 \mathrm{~mW}, 15 \mathrm{~min}$ ) of the entire imaging field and corresponding temporal evolutions (c and d, $\Delta t=432 \mathrm{~ms}$ ) of the integrated emission intensity.

illumination of a defined region of interest at $\lambda_{\mathrm{Ac}}$, with concomitant detection of the light emitted from the same area (a in Fig. 5), permits the acquisition of a temporal profile of the fluorogenic transformation. The resulting plot (c in Fig. 5) shows an increase in the emission of the fluorescent product with saturation reached after $c a .15 \mathrm{~min}$. The time $\left(t_{50}\right)$ required to convert $50 \%$ of 1 into 2 is $294 \mathrm{~s}$ under these experimental conditions.

The wavelength employed to activate 1 and excite 2 in PBMA happens to be positioned within the surface-plasmon band of silver nanoparticles (Fig. S4, ESI $\dagger$ ). ${ }^{13 a}$ As a result, these particular metal nanostructures can have a pronounced influence on the photochemical transformation of $\mathbf{1}$ into 2 as well as on the photophysical behavior of the product. Indeed, a remarkable increase in fluorescence (b in Fig. 5) is actually observed upon activation of a polymer film deposited on a glass slide pre-coated with silver nanoparticles. $\ddagger$ Under these conditions, the emission intensity, integrated within the activated area, is 6.0 times greater than that detected (a in Fig. 5) in the absence of metal nanostructures. This value is consistent with the ratio between the emission intensities recorded for 2 in PBMA with and without silver nanoparticles (Fig. S4, ESI $\dagger$ ), which is 5.6. Thus, the interaction of the exciting radiations with the surface plasmons of the metal nanostructures clearly enhances the emission intensity of the fluorescence product. The local increase in electromagnetic field facilitates the excitation of proximal fluorophores. Additionally, the nanoparticles accelerate the radiative deactivation of the excited molecules. In fact, the fluorescence of 2 decays biexponentially also in the presence of the metal nanostructures with a $\tau_{1}$ of $0.15 \mathrm{~ns}(86 \%)$ once again, but $\tau_{2}$ decreases to only $0.79 \mathrm{~ns}(14 \%)$. Similarly, the very same plasmonic effects appear also to accelerate the photochemical reaction. In the presence of the nanoparticles, the temporal evolution of the emission intensity (d in Fig. 5) reaches saturation after only ca. 5 min and the corresponding $t_{50}$ shortens to $66 \mathrm{~s}$.

The ability of the silver nanoparticles to facilitate the photoinduced conversion of a nonemissive species into a fluorescent

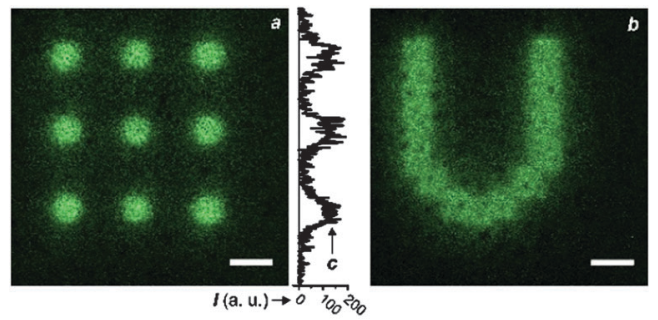

Fig. 6 Confocal laser-scanning fluorescence images ( $a$ and $b, \lambda_{E x}=405 \mathrm{~nm}$, $\lambda_{\mathrm{Em}}=430-650 \mathrm{~nm}$, scale bar $\left.=30 \mu \mathrm{m}\right)$ of PBMA films, doped with $1(3 \% \mathrm{w} / \mathrm{w})$ and deposited on glass slides with silver nanoparticles on their surface, recorded after illumination ( $\left.\lambda_{\mathrm{Ac}}=405 \mathrm{~nm}, 0.1 \mathrm{~mW}, 300 \mathrm{~s}\right)$ of defined areas within the imaging field. Profile of the emission intensity (c) measured across some of the activated areas in a.

one and to enhance the emission of the latter can be exploited to write and read microstructures with visible light at relatively low illumination intensities. Arrays of dots (a in Fig. 6) with a diameter of $c a .20 \mu \mathrm{m}$ or even letters (b in Fig. 6) with microscaled dimensions can be imprinted on a PBMA film, doped with 1, under the influence of an activating laser and then read by measuring the fluorescence of 2 photogenerated in the illuminated areas. Furthermore, the lack of any diffusion of the fluorescent species within the polymer matrix preserves the geometry of the written pattern and permits their optical reading, even after relatively long times. Specifically, the imprinted information can be read $c a$. 320 consecutive times before the signal-to-background ratio decreases from infinite to 1.25.

In summary, our results demonstrate that the covalent connection of a carbazole fluorophore to a photoresponsive oxazine permits the activation of the emission of the former with the photoinduced cleavage of the latter. This photochemical transformation is facilitated if the carbazole-oxazine dyads are immobilized within a polymer matrix in proximity to silver nanoparticles. The plasmonic effects responsible for promoting the activation of the reactant cause also a pronounced enhancement in the emission of the product. As a result, microscaled fluorescent patterns can be generated and detected efficiently with far-field optics at relatively low illumination intensities. Thus, these operating principles can lead to the development of strategies for the optical writing and reading of information based on metallic nanostructures and photoactivatable fluorophores. In addition, the activated fluorescence of this system can be exploited to monitor conveniently the influence of plasmonic effects on photochemical reactions. Therefore, such structural design can evolve into valuable molecular probes to investigate the fundamental factors regulating the influence of metallic nanostructures on the excitation dynamics of organic chromophores.

JGA is grateful for a Beatriu de Pinós postdoctoral grant from the Generalitat de Catalunya (Spain, grant 2011 BP-A-00270). FMR acknowledges the NSF (CHE-1049860) for financial support.

\section{Notes and references}

$\ddagger$ AFM images (Fig. S6, ESI $\dagger$ ) show that the average diameter of the nanoparticles is $c a .20 \mathrm{~nm}$.

1 L. M. Wysocki and L. D. Lavis, Curr. Opin. Chem. Biol., 2011, 15, 752-759. 
2 D. Puliti, D. Warther, C. Orange, A. Specht and M. Goeldner, Bioorg. Med. Chem., 2011, 19, 1023-1029.

3 W.-H. Li and W.-H. Zheng, Photochem. Photobiol. Sci., 2012, 11, 460-471.

4 P. Klán, T. Šolomek, C. G. Bochet, A. Blanc, R. Givens, M. Rubina, V. Popik, A. Kostikov and J. Wirz, Chem. Rev., 2013, 113, 119-191.

5 F. M. Raymo, Phys. Chem. Chem. Phys., 2013, 15, 14840-14850.

6 Y. Xu, T. J. Melia and D. T. Toomre, Curr. Opin. Chem. Biol., 2011, 15, 822-830.

7 F. M. Raymo, ISRN Phys. Chem., 2012, 619251.

8 S. van de Linde, M. Heilemann and M. Sauer, Annu. Rev. Phys. Chem., 2012, 63, 519-540.

9 T. Ha and P. Tinnefeld, Annu. Rev. Phys. Chem., 2012, 63, 595-617.

10 R. A. Alvarez-Puebla, L. M. Liz-Marzán and F. J. García de Abajo, J. Phys. Chem. Lett., 2010, 1, 2428-2434.

11 H. Nabika, M. Takase, F. Nagasawa and K. Murakoshi, J. Phys. Chem. Lett., 2010, 1, 2470-2487.
12 J. C. Scaiano and K. Stamplecoskie, J. Phys. Chem. Lett., 2013, 4, 1177-1188.

13 (a) E. R. Thapaliya, S. Swaminathan, B. Captain and F. M. Raymo, J. Am. Chem. Soc., 2014, 136, 13798-13804; (b) E. R. Thapaliya, B. Captain and F. M. Raymo, Asian J. Org. Chem., 2015, 4, 233-238.

14 J. Garcia-Amorós, S. Swaminathan, S. Sortino and F. M. Raymo, Chem. - Eur. J., 2014, 20, 10276-10284.

15 X. Ragàs, A. Gallardo, Y. Zhang, W. Massad, C. D. Geddes and S. Nonell, J. Phys. Chem. C, 2011, 115, 16275-16281.

16 D. S. Silvester, A. J. Wain, L. Aldous, C. Hardacre and R. G. Compton, J. Electroanal. Chem., 2006, 596, 131-140.

17 N. R. Armstrong, R. K. Quinn and N. E. Vanderborgh, Anal. Chem., 1974, 46, 1759-1764.

18 L. J. Núñez-Vergara, J. A. Squella, C. Olea-Azar, S. Bollo, P. A. Navarrete-Encina and J. C. Sturm, Electrochim. Acta, 2000, 45, 3555-3561. 